\title{
Research on Green Degree Evaluation of Manufacturing Reverse Logistics
}

\section{Ting Tian, Yaping Chen}

Faculty of Management, Shanghai University of Engineering Science, Shanghai, China. Email: TTingdream@163.com

Received December $12^{\text {th }}, 2013$; revised January $12^{\text {th }}, 2014$; accepted January $19^{\text {th }}, 2014$

Copyright (C) 2014 Ting Tian, Yaping Chen. This is an open access article distributed under the Creative Commons Attribution License, which permits unrestricted use, distribution, and reproduction in any medium, provided the original work is properly cited. In accordance of the Creative Commons Attribution License all Copyrights @ 2014 are reserved for SCIRP and the owner of the intellectual property Ting Tian, Yaping Chen. All Copyright (C) 2014 are guarded by law and by SCIRP as a guardian.

\section{ABSTRACT}

The contradiction between economic development and environmental deterioration makes people begin to have a further understanding of the ecology and environment. To promote effective development of reverse logistics and implementation, according to general activity process of manufacturing enterprises, this paper builds the manufacturing system model of reverse logistics. On the basis of activities in this system used to find out the main factors which influence the green degree evaluation and promote the forming of a green degree evaluation index system, it will provide related enterprises with a detection method for reverse logistics performance evaluation with the fuzzy analytic hierarchy process (FAHP) and fuzzy comprehensive evaluation method for the construction of the test system.

\section{KEYWORDS}

\section{Manufacturing Industry; Reverse Logistics; Green Degree; Fuzzy Evaluation}

\section{Introduction}

With arrival of the new business model, supply chain system, the reverse logistics which is opposite to forward logistics activities comes into people's horizons. From the microcosmic point of view, the reverse logistics has an explicit and implicit effect on improving customer value, enhancing the competitive advantage, reducing the material cost, increasing the profit of the enterprise, improving the environment behavior and shaping the corporate image. From a macro point of view, the reverse logistics is conducive to the rational flow of social resources, which help save resources and improve the sustainable development of environment and economy, etc. Therefore, the implementation of the "reverse logistics" conforms to the requirements of The Times.

18th CPC National Congress takes "ecological civilization" to the strategic height. The reverse logistics which is closely connected with environment and ecology will become the focus of developing and exploring in the industry. At the same time, reverse logistics is based on circular economy and sustainable development which become an important area of improving enterprise competitive advantage and management efficiency. At present, the loss from returns, overproduction, nonconforming returns, scrapping and damage is growing at an alarming rate every year. There is an appealing prospect in carrying out reverse logistics for a great resource and environmental constraints of China.

In a narrow sense, the "green degree" refers to the degree of impact of the enterprises' all production and business operation activities on the environment. A broader understanding refers to the comprehensive evaluation of green products, economy and technology. The smaller the impact of production and business operation activities on the environment, the higher "green degree" in the enterprise, but the lower conversely [1].

In the quantitative research on the reverse logistics, we introduced the concept of green degree for facilitating the quantification and evaluation of reverse logistics on the environment pollution. Green degree can be understood as the degree of the sustainable development of reverse logistics, or its friendly degree of the ecological envi- 
ronment. It is the comprehensive evaluation index of economy, society and environment in the harmony degree.

In the research about the green degree evaluation, Shan Hong and Yao Fangyuan [2] put forward the green degree evaluation formula, $G=f(T, E, C, t)$. $G$ refers to the system of green logistics, and $\mathrm{T}$ refers to the advanced logistics technology. It consists of various logistics technical indicators, and E refers to the environment coordination, It is made up of various coordination environmental parameters. $\mathrm{C}$ points to economic rationality. It is made up of various economic indicators. $\mathrm{T}$ is the time. $\mathrm{F}$ is the increasing function of time t. Wang Shuo, Gong Daning [3] try to set up green degree evaluation index system of green virtual enterprise and dimensionless index numerical process through the interpretations of the meanings of the evaluation index and efficacy coefficient method. Then analysis and evaluation experts build a comprehensive evaluation model of green degree in green virtual enterprise. It provides the theory and practice basis for tracing and monitoring the efficacy of the virtual enterprise operation. Based on social benefits, Jiang Mingxia [4] constructs a scientific and reasonable general social benefits performance evaluation system which includes social responsibility, the social image, harmonious and unified social contribution linked with economic benefits which should be placed in the development of the strategic position. In manufacturing enterprises, Li Hui [5] puts forward the green degree evalua- tion of manufacturing enterprise production logistics system based on the analysis of the characteristics of manufacturing. By using the thought of system engineering, he establishes the mathematical model, the integrated use of fuzzy mathematics, the matter-element model and AHP to do the evaluation from the aspects of environmental performance, resource performance, economic performance and technical performance. However, the green degree evaluation of reverse logistics in manufacturing industry has not been studied in the corresponding paper. This article embarks from the manufacturing and analyzes the green degree evaluation system through the building of structure model of the reverse logistics.

\section{The Structure Model of Reverse Logistics in Manufacturing}

The manufacturing reverse logistics is a series of process from product design and research to processing and manufacturing, and finally flow into consumers' hands. And for different reasons, the products get into the maintenance, recycling centers. At this time, the $3 \mathrm{R}$ (reduce, reuse, and recycle) principle is throughout the whole process. That would form a "resource - consumption - renewable resources" closed-loop feedback process. Under the support of ecological civilization, sustainable development and recycling economy theory the reverse logistics achieve enterprises, consumers and the government's participation and support. We can see the details from Figure 1.

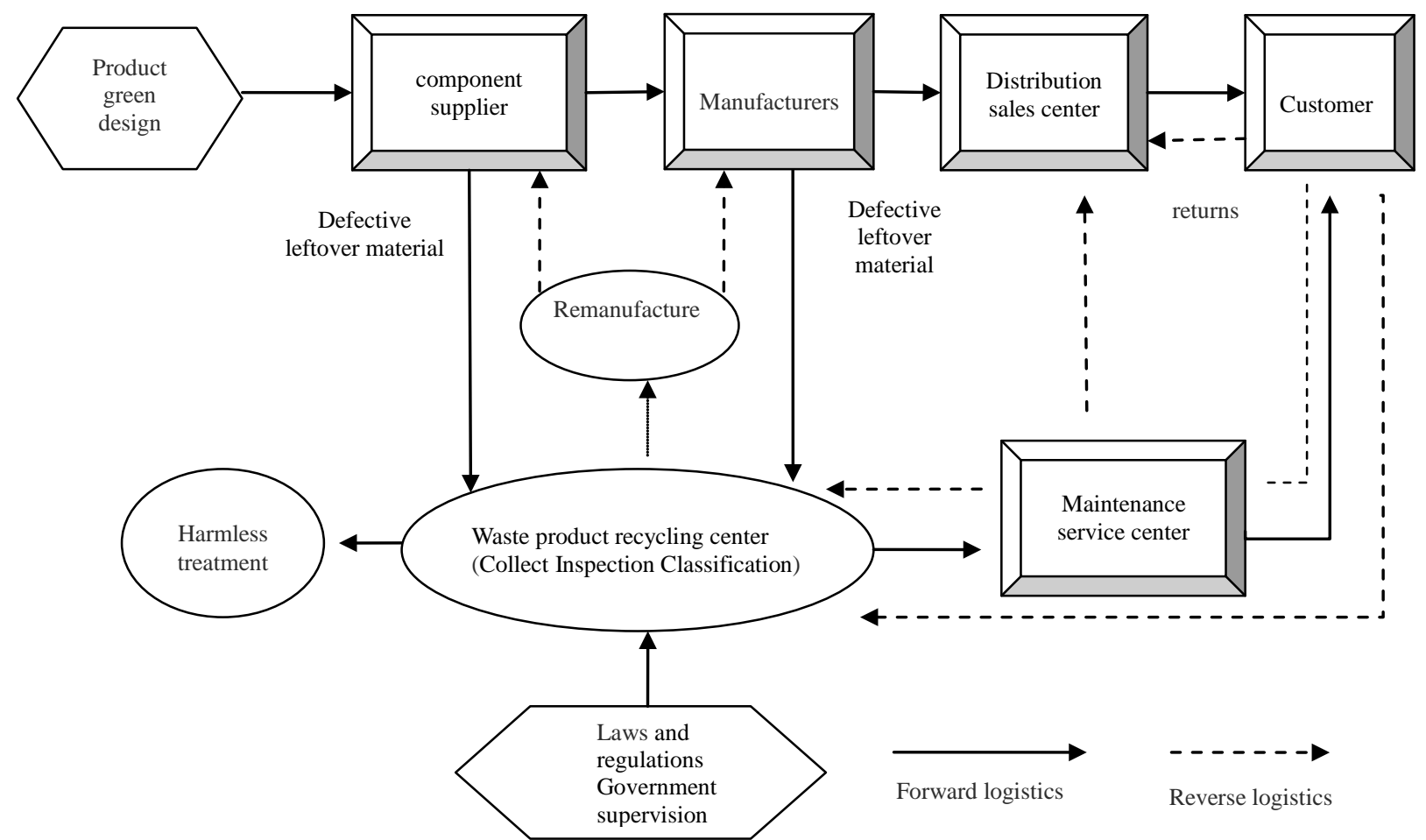

Figure 1. Manufacturing model of the reverse logistics system. 


\subsection{Implementation Phase and Principle}

In the stage of product design and development, we should follow the principle of reduction, the concept of green design; In the process of production, with sustainability as the creed, the defective leftover material sent to the recycling center recycle; From consumers to the service center or directly to the waste product recycling center stage, through the detection and classification, repair and remanufacturing of waste resources for meticulous excavation, which always reflect the principle of "reuse, recycle".

\subsection{Logistics Functions and Participants}

Reverse logistics system functions include recycling, detection, classification, and then according to the different functions for recycling and remanufacturing. Manufacturing reverse logistics is closely relationship between these functions which linked together closely to maximize the use of products and resources. In the whole process of function implemented, the government gives the guidance of laws and regulations, and policy support. Parts suppliers, manufacturers, distributors and repair and recycling center in the active cooperation of customers, based on the characteristics of their products and services, are participate in the green product and recycling activities.

\section{The Construction of Evaluation Index System}

Before manufacturing reverse logistics green degree evaluation, we should first analyze its related factors. In order to build the index system has better scientific nature and operability, while guarantee the wholeness and completeness of indicators. This paper mainly analyzes the factors which influence reverse logistics in manufacturing activity, and then classified and stratified them to different angles. Finally classified as environmental in- dicators, resources indicators, economic indicators, technical index and social index (as shown in Figure 2), then make more detailed analysis on the five indexes.

\subsection{Environmental}

All stages of Manufacturing reverse logistics activities will have different influence on the environment. The design of environmental index directly reflects the friendly degree of each factor to the environment. These specific factors in logistics activity process include air pollutants (such as $\mathrm{CO}_{2}, \mathrm{SO}_{2}$, $\mathrm{PH} 2.5$ ), solid wastes (all kinds of harmful substances and soluble highly toxic waste residues may contain such as mercury, yellow phosphorus, etc.), water pollution (lubricating oil, acid, phenol compounds, etc.), noise pollution (divided according to the level of noise $\mathrm{dB}$ ) and so on.

\subsection{Resource}

Resources index in green degree examination of manufacturing reverse logistics mainly includes the use of raw materials and energy resources. The slower raw material consumption and smaller the consumption, the better the performance of resource recycling system. Meanwhile, the effectiveness of overall recycling process of waste products would be reflected by the use recycle material utilization, product recovery and remanufacturing rate.

\subsection{Economic}

The reverse logistics operation for the whole product life cycle runs throughout the whole supply chain system. This paper focuses on the enterprise costs, user costs and ecological costs. Enterprise costs include the design, manufacture, storage, distribution and after-sale service cost; The user costs mainly contains the user's operation and maintenance costs; Ecological costs include pollution charges, pollution control, and the disposal of scrap products.

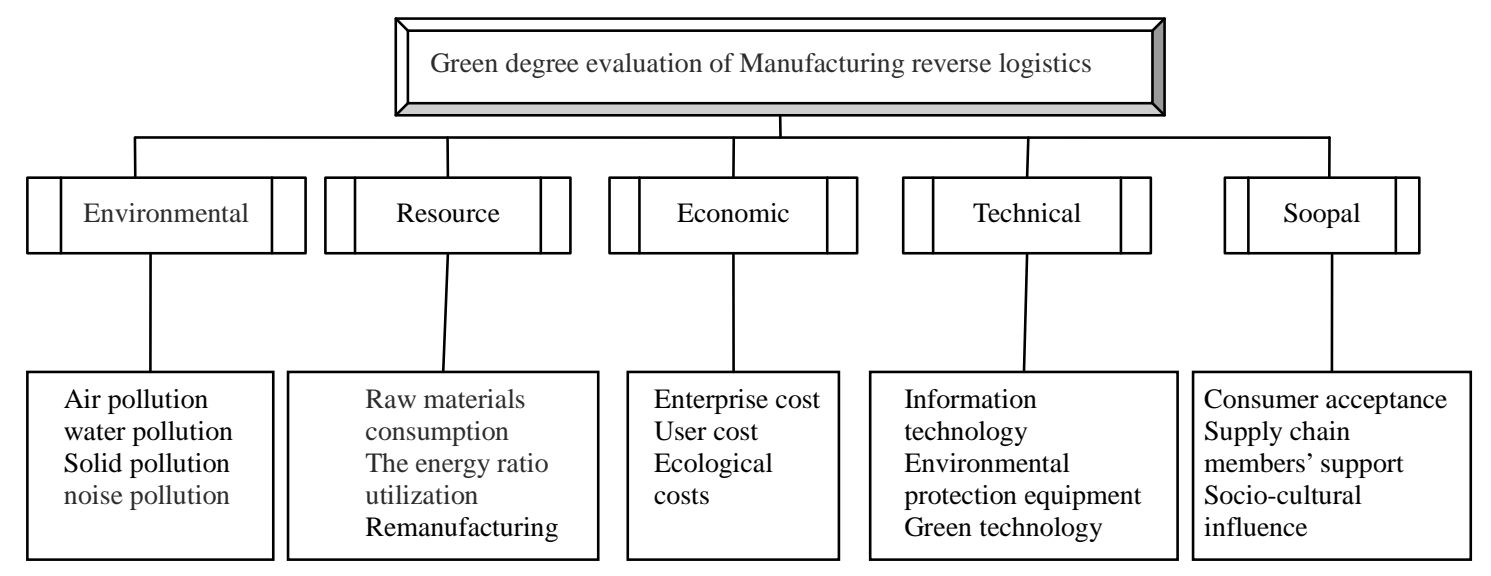

Figure 2. Green degree evaluation system of manufacturing reverse logistics. 


\subsection{Technical}

Technical indicators are used to evaluate the advancement of technology in reverse logistics system. Modern logistics is a new kind of industry based on information technology and intelligent technology. More technical support is needed to the integration between reverse logistics and forward logistics. The technical performance can be examined by the information management system, the use of environmental protection equipment and the implementation of green technology. We can reduce the resource consumption and environmental damage through the effective use of advanced technology in the process of reverse logistics.

\subsection{Social}

As the reverse logistics activities, the defective products in consumers' hands return to the manufacturers, the waste product flow to the recycling center. Manufacturers and recycling center not only reduce product damage and the damage to the environment through certain processing technology, but also can increase the economic benefit and establish a good corporate image. We measure the social indicators based on the consumer identity, supply chain members' support and the effect of social culture.

\section{Green Evaluation Based on FAHP}

After the building of green degree evaluation index system in manufacturing Reverse logistics, we need a certain method to evaluate the index system. To accord with the fuzziness and subjectivity of people thinking, people introduced to the fuzzy thought and methods to analytic hierarchy process (AHP), and form the fuzzy analytic hierarchy process (FANP) which based on fuzzy complementary judgment matrix. On the one hand, to determine the weights of evaluation indexes with FAHP accord with the objectivity of the problem and the subjectivity of people thinking; on the other hand, the assessment of green level in manufacturing enterprises with FAHP can avoid absoluteness of experts and make a fast and accurate decision.

\subsection{To Determine the Fuzzy Evaluation Index and Evaluation Sets}

Setting up the fuzzy evaluation index set $\mathrm{X}=\left(\mathrm{X}_{1}, \mathrm{X} 2\right.$, $\mathrm{X} 3, \mathrm{X} 4)$ According to the evaluation index system in figure 2 . Then subdivide the various indicators of $X$, such as $X_{1}$ is environmental indicators. The environmental indicator has four factors, so $X_{1}=\left(X_{11}, X_{12}, X_{13}, X_{14}\right)$. Other similar factors could express as $\mathrm{X}_{\mathrm{i}}=\left(\mathrm{X}_{\mathrm{i} 1}, \mathrm{X}_{\mathrm{i} 2}, \cdots, \mathrm{X}_{\mathrm{in}}\right)$

This article divided evaluation level into $4, \mathrm{~V}=\left(\mathrm{V}_{1}\right.$, V2, V3, V4) = (A. B. C. D)

\subsection{The Weight Analyze of Evaluation Indexes}

1) Through the score of each index and factor from evaluation experts to build the fuzzy complementary matrix $\mathrm{P}=\left(\mathrm{p}_{\mathrm{ij}}\right)_{\mathrm{n} \times \mathrm{n}}$ and here:

$$
\mathrm{p}_{\mathrm{ij}}+\mathrm{p}_{\mathrm{ji}}=1, \mathrm{i}, \mathrm{j}=1,2 \cdots \mathrm{n}
$$

The weight $\mathrm{W}$ can be expressed as

$$
\mathrm{w}_{\mathrm{i}}=\frac{1}{\mathrm{n}}-\frac{1}{2 \alpha}+\frac{2}{\mathrm{n} \alpha} \sum_{\mathrm{k}-1}^{\mathrm{n}} \mathrm{p}_{\mathrm{ik}} \quad \alpha=\frac{\mathrm{n}-1}{2}
$$

2) Single-level sorting and consistency check. We can give a critical value $\varepsilon>0$ to make the consistency check of fuzzy judgment matrix P. when $\rho<\varepsilon$ we can say matrix $\mathrm{P}$ has the satisfactory consistency; or matrix $\mathrm{P}$ has a large deviation from consistency degree and needs the progressive correction. In practice, the value can be appropriately selected depending on the circumstances. The smaller the value of $\varepsilon$, the higher the requirement for evaluation experts; Otherwise the lower requirements.

According to the equation:

$$
\begin{aligned}
R & =\left(r_{i j}\right)_{n \times n}=\left(\frac{1}{n} \sum_{k=1}^{n}\left(p_{i k}-p_{j k}\right)+0.5\right)_{n \times n} \\
C & =\left(c_{i j}\right)_{n \times n}=\left(p_{i j}-r_{i j}\right)_{n \times n}
\end{aligned}
$$

So the consistency check value:

$$
\rho=\frac{2}{n(n-1)} \sum_{1 \leq i \leq j \leq n}\left|C_{i j}\right|
$$

3) We can get the total weight order of $X_{1}, X_{2}, X_{3}, X_{4}$, $\mathrm{X}_{5}$ from top to bottom layer by layer according to the weight of evaluation index calculated in part 2)

\subsection{Fuzzy Synthetic Evaluation}

Making the evaluation for all levels of index and the factors within indicators with Experts Grading Method, namely, get the judgment of every index is A, B, C or D, then forming the corresponding judgment matrix $G_{i}$. Due to different rows in the $G$ reflects the membership degree of each level indicators from a different point of the single factor. Combine the Fuzzy weight matrix with different $\mathrm{G}$, we can obtained the degree of membership of each level indicators of evaluated objects from a general point. We can call it $\mathrm{D}$, the fuzzy comprehensive evaluation results. Finally we get the results of green degree evaluation in manufacturing enterprise reverse logistics

$$
D=W^{*} G=\left[W_{1}, W_{2}, W_{3}, W_{4}, W_{5}\right]\left[\begin{array}{c}
G_{1} \\
G_{2} \\
G_{3} \\
G_{4} \\
G_{5}
\end{array}\right]
$$


According to the results of the analysis of the matrix D, we can know the implementation of green degree in enterprise reverse logistics and make a categorization.

\subsection{Case Study}

We select one electrical appliances manufacture enterprise reverse logistics system as the evaluation objects. By the above method we get the weight of the five indicators $\mathrm{W}=(0.2,0.3,0.25,0.1,0.15)$. Then we get the judgment matrix $\mathrm{Gi}=(83 \%, 60 \%, 65 \%, 70 \%, 84 \%)$ vie three experts' grading. So the fuzzy comprehensive evaluation results:

$$
\mathrm{D}=\mathrm{W}^{*} \mathrm{G}=[0.2,0.3,0.25,0.1,0.15]\left[\begin{array}{c}
83 \% \\
60 \% \\
65 \% \\
70 \% \\
84 \%
\end{array}\right]=0.7045
$$

The result shows that the enterprise recovery system has some problems on the utilization of resources, technology and equipment. They should make further modification according to the lower numerical value of index.

\section{Conclusions}

With the rapid development of economy, people have high expectations for the environment. The laws and regulations of environmental protection on enterprise behavior become stricter. Environmental performance has gradually become an important index in enterprise operating performance evaluation. However, the related policies and facilities on reverse logistics in China's manufacturing industry are not yet in place, and the enthusiasm of enterprises in reverse logistics is not enough. Green degree evaluation is helpful for the public to have a comprehensive understanding of reverse logistics from all walks of life. What's more, it can promote a rapid development of reverse logistics.

But there are too many influence factors about green degree evaluation of manufacturing reverse logistics and the emphasis of the companies is different. Therefore, the construction of evaluation index needs to be properly changed in the face of the different enterprises, so that it could be better to check the data or results we need, and then make more scientific and more reasonable suggestions for the green development of enterprises.

\section{Acknowledgements}

This work was financially supported by Scientific Research \& Innovation Project under Shanghai Municipal Educational Committee (12ZS174) and Scientific Research Innovation Program (A-0903-13-01058) of Shanghai University of Engineering Science.

\section{REFERENCES}

[1] L. J. Zhao, "Research on the Evaluation of Environmental Logistics Based on Fuzzy Comprehensive Evaluation,” Journal of Wuhan University of Technology, Vol. 30, No. 6, 2008, pp.174-177.

[2] H. Shan and F. Y. Yao, "The Green Degree Evaluation of the Logistics System,” Reform \& Opening, Vol. 18, 2009, pp. 76-78.

[3] S. Wang, D. N. Gong, "The Green Degree Valuation System of Green Virtual Enterprise,” Hefei University of Technology, Vol. 21, 2007, pp. 31-34.

[4] M. X. Jiang, "Performance Evaluation System on Social Benefits of Enterprise Reverse Logistics System,” Business Economy, Vol. 10, 2011, pp. 34-36.

[5] H. Li, "Green Degree Assessment of the Production Logistics in Manufacture,” M.S. Thesis, Shandong University of Science and Technology, Jinan, 2006. 